\title{
Cyclosporin A Reversed Chemoresistance of a Patient with Pure Red Cell Aplasia Secondary to Thymoma
}

\author{
PENG LI ${ }^{1}$, FENGCAI DU ${ }^{2}$, ZHAOHUA GONG ${ }^{1}$, BAOHONG HU ${ }^{1}$, \\ CHENG CHI $^{1}$, HONGJIN CHU ${ }^{3}$ and JIAN CHEN ${ }^{1,3}$ \\ ${ }^{1}$ Department of Oncology, the Affiliated Yantai Yuhuangding Hospital of Qingdao University, Yantai, P.R. China; \\ ${ }^{2}$ The First Clinical College of DaLian Medical University, DaLian, P.R. China; \\ ${ }^{3}$ The Central Laboratory, the Affiliated Yantai Yuhuangding Hospital of Qingdao University, Yantai, P.R. China
}

\begin{abstract}
Case Report: This case study reports on a patient who relapsed with thymoma (mixed type) nine years after tumor resection. After four courses of rescue chemotherapy (docetaxel and cisplatinum), the patient was further diagnosed with pure red cell aplasia. It was noticed that cyclosporin A (CsA), which was administered to treat aplasia, could reverse chemoresistance. Its mechanism is not completely clear, but the hypothesis of CsA inhibiting Pglycoprotein mediated drug efflux is the most acceptable.
\end{abstract}

\section{Case Report}

Setting. Pure red cell aplasia (PRCA) is a rare hematological disease characterized by selective decrease in erythrocytes and their progenitors in the peripheral blood and bone marrow respectively (1). This disease occurs occasionally as a complication of myasthenia gravis (MG) without thymoma or, as in this case, the patient presented with PRCA secondary to thymoma; and immunosuppressive agents are usually employed for treatment in addition to blood transfusion (2). Thymoma is the most common thymic epithelial tumor, of which completeness of resection is a critical factor for determining recurrence and overall survival in early stage thymoma. Adjuvant chemotherapy, however, is mostly reserved for advanced stage thymomas. However, relapse of thymoma is not rare, with approximately $80 \%$ of the patients who have achieved remission relapsing within two years of treatment (3).

Cyclosporin A (CsA) is an immuno-suppressor that has been used widely to prevent rejection in organ transplantation. In recent years, some in vitro studies have revealed that CsA

Correspondence to: Dr. Jian Chen, the Affiliated Yantai Yuhuangding Hospital of Qingdao University, Yantai, Shandong, 264000, P.R. China. Tel: +86 18660086778, e-mail: chenjianyt@163.com

Key Words: Cyclosporin A, chemoresistance, thymoma. has the potential to reverse chemoresistance by inhibiting Pglycoprotein (P-gp)-mediated drug efflux or through other unknown mechanisms $(4,5)$. Here, we report that the development of chemoresistance of a patient with PRCA secondary to thymoma (mixed type) was reversed by CsA.

Patient details. A 47-year-old male underwent extensive thymectomy with invaded tissue and lymph node dissection through a middle thoracotomy nine years ago. The histologic diagnosis was mixed thymoma (type $\mathrm{AB}$ ) showing both spindle and oval cells. No further treatment such as chemotherapy or radiation therapy was given.

Treatment. The patient was admited to the Affiliated Yantai Yuhuangding Hospital of Qingdao University on June 5th, 2013. The following symptoms were recorded at admission: swollen facial and chest area, varicose veins, eyelid edema and exophthalmos. Retinal examination showed retinal edema and venous congestion. Other complaint of discomfort included headache, dizziness and tinnitus. Compared with patient's previous scan on Oct 16th, 2012 (Figure 1), this patient's chest computerized tomography (CT) (Figure 2) showed a large tumor cell embolus in the left brachiocephalic, superior vena cava and right atrium; a tumor mass $(5.5 \mathrm{~cm} \times 4.1 \mathrm{~cm})$ at the right anterior mediastinum; intrapulmonary nodular shadow and shadows around the hepatic hilar region and coeliac trunk were notable. These suggested thymoma relapse. Bone scanning showed abnormal diffused sternal radioactive concentration.

After four courses of chemotherapy. $120 \mathrm{mg}$ docetaxel i.v. d1 (H20030540, Aventis Pharma, French) and $40 \mathrm{mg}$ cisplatinum i.v. d2-4(H21020751, JIUTAI Pharmaceutical, China), chest CT (Figure 3) showed minor tumor progression and clinical evaluation determined that the disease was stable. Blood routine examination on Sept 24th, 2013 revealed a red blood cell count of $1.21 \times 10^{12} / 1$, hemoglobin $65 \mathrm{~g} / 1$, platelet $198 \times 10^{9} / 1$ and an erythrocyte sedimentation 
rate of $3.49 \times 10^{9} / 1$. Other biochemical data such as blood urea, electrolytes and liver function were normal. Bone marrow aspirate (Figure 4) revealed an extremely low fraction of erythrocytes with no abnormalities in mature red blood cell formation, nor dysplasia of leukocytes or platelets, which yielded to PRCA. Then, $5 \mathrm{mg} / \mathrm{kg}$ of cyclosporin A (H20090495 Novartis Pharma GmbH, Germany) was given once a day along with $30 \mathrm{mg}$ of prednisone.

The patient was then given a further four courses of chemotherapy: $100 \mathrm{mg}$ docetaxel i.v. d1 (H20030540, Aventis Pharma, French), $30 \mathrm{mg}$ cis-platinum iv d2-4 (H21020751, JIUTAI Pharmaceutical, P.R. China).

After four cycles of this combination, the chest CT on May 25th, 2014 (Figure 5) showed significant tumor regression compared with the previous CT scan. Clinical efficiency evaluation was determined as partial regression (PR). No further treatment beside oral administrated prednisone was carried out until June 2015 when another chest CT revealed progressed thymoma (PD) (Figure 6). Bone marrow cell morphology examination (Figure 7) on June 30th, 2015 revealed that PRCA was stable (SD). The patient died on 5th January 2016.

\section{Discussion}

Thymoma is one of the most frequent neoplasms in the anterior mediastinum of adult patients. Based on the appearance of neoplastic epithelial cells, thymoma has been classified as type A (spindle or oval cells), type B (dendritic or epithelioid cells), and type $\mathrm{C}$ (exhibiting combined features of types A and B) according to the World Health Organization (WHO) (6). Myasthenia gravis, lambert-Eaton myasthenic syndrome, pemphigus, subacute sensory neuronopathy, pure red cell aplasia, and immunodeficiency are paraneoplastic syndromes of thymoma $(2,7)$.

PRCA is one of the most remarkable associations characterized by erythropoietic failure with preserved granulopoiesis and megakaryopoiesis. Although PRCA occurs in only $5 \%$ of thymoma patients, it is usually associated with poor prognosis (8). Murakawa et al. (2002), reported that two out of three patients who developed PRCA following thymectomy died within one year (2). Surgical removal or debulking of the tumor mass are the main treatments of thymoma. Complete of tumor resection improves patients' long-term prognosis (9). In this reported case, the patient also obtained a long time Progression-FreeSurvival (FPS) (nine years) since surgical removal of the thymoma. Combined therapy of surgery and remission induction therapy with CsA was recommended for the patient with a thymoma associated with PRCA, and obtained a relatively good clinical response.

Drug resistance is the main obstacle that often develops during chemotherapy for cancer. It occurs mainly due to reducing intracellular drug accumulation, preferentially by $\mathrm{ABC}$ transporters, which affects modulation of the activity of MRP1 and P-gp, and stimulation of MAPKs cell signaling pathways (10). CsA blocks the function of the MDR pump and modulates the resistance to anticancer drugs (11). A study by Goldberg et al. (1988), showed that CsA accumulation was reduced in multidrug-resistant CHRC5 $\mathrm{CHO}$ cells with high levels of P-gp expression, and vinblastine accumulation increased in resistant cell lines after treatment with CsA (12). Mutch et al. (1992), found that CsA sensitized resistant ovarian cancer cells to cisplatin which is not a P-gp substrate and is not significantly transported in the form of a glutathione conjugate by other transporters (13). However, this drug pump- and $\mathrm{ABC}$ transporter-independent Cyp-mediated chemoresistance is not easily explained.

A number of cell experiments have provided evidence of the effect of CsA modulating the resistance to anticancer drugs. Mutch et al. (1992), evaluated the potential of CsA to decrease drug resistance in ovarian cancer cells selected for resistance to cisplatin (2780-CDDP cells). The study showed that CsA significantly and selectively increased the sensitivity of A2780-CDDP cells to cytolysis by CDDP but not basal resistance in the CDDP-sensitive parent cells (A2780) (13).

Contrary to the promising preclinical in vitro studies, clinical studies combining chemotherapeutics and CsA showed low anticancer activity. In the study of Morjani et al. (2010), the combination of CsA and cisplatin was investigated in 26 patients with recurrent epithelial ovarian cancer (14). The patients with measurable lesions which were resistant to cisplatin received this combination. Only a single patient had a complete response, with two experiencing a partial response to the combination. This indicates little effect of CsA in reversing cisplatin resistance. In another study, the response rate of a $26 \mathrm{~h}$ continuous infusion with CsA combined with carboplatin and $\alpha$ interferon was tested in recurrent ovarian cancer (15). Three of thirty patients had partial response and nine were stable for $>4$ months. These results revealed that the reversal of clinical drug resistance by CsA was too low to warrant further investigation. Until now, there have not been sufficient clinical studies to support the function of CsA for modulating resistance to chemotherapy.

In this case, we report on a patient with PRCA secondary to thymoma (mixed type) whose chemoresistance was reversed by CsA administration. The comparison of chest CT in June 5, 2013 and Dec 24, 2013 showed limited clinical efficiency of the docetaxel and cis-platinum regimen, which indicated development of chemo-resistance during four cycles of treatment. Chest CT after six months on May 25th, 2014 revealed better clinical efficiency of the four courses of CsA combined with chemotherapy (CsA and prednisone 


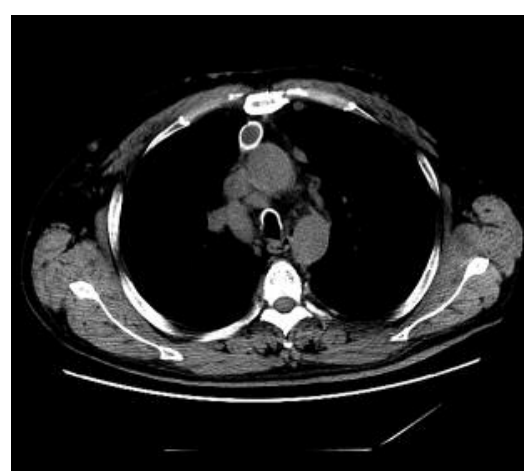

Figure 1. Chest CT showing steady disease after thymoma (malignant mixed type) resection on Oct 16th, 2012.

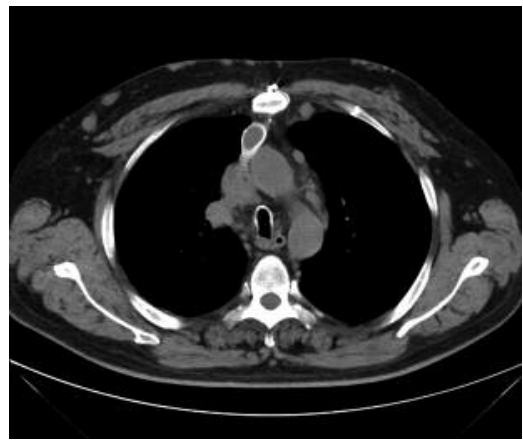

Figure 2. Chest CT showing tumor cell embolus in the left brachiocephalic, superior vena cava and right atrium on June 5th, 2013.

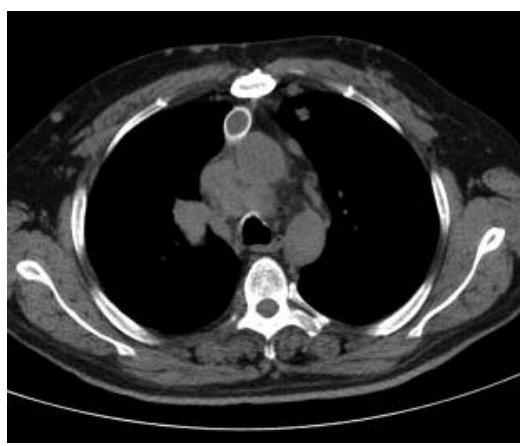

Figure 3. Chest CT showing therapeutic evaluation of 4 courses of chemotherapy was PD on Dec 24th, 2013.

combined with docetaxel and cisplatinum). CsA was originally used to treat PRCA, but evident clinical outcome revealed that potential drug resistance can be reversed by the function of CsA. However, chest CT a year later (no chemotherapy during this time) showed relapse of thymoma. No chemotherapy was given for one year, during which time oral administration of CsA was taken by the patient, and it

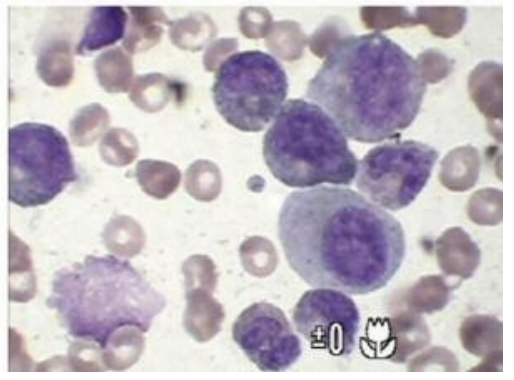

Figure 4. Bone marrow cell morphology examination showing over proliferation in more than $80 \%$ of all granulocytes; suppressed erythroid without immature polychromatic erythrocyte and few polychromatic erythrocytes on Sept 25th, 2013.

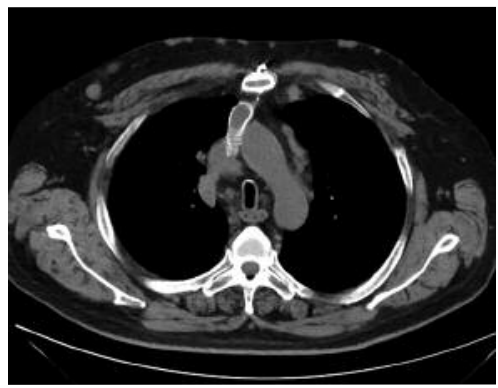

Figure 5. Chest CT showing tumor regression at the mediastinum compared to last CT scan. Clinical efficiency evaluation was determined as partial regression on May 25th, 2014.

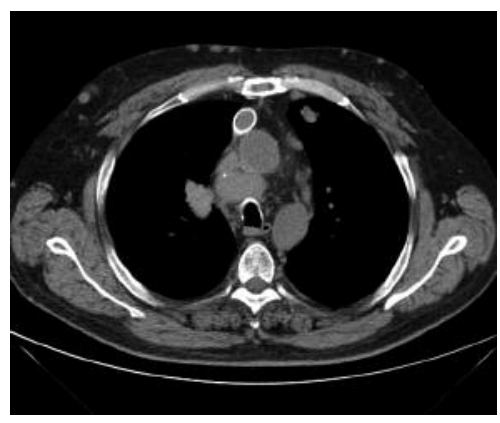

Figure 6. Chest CT showing relapse of thymoma after one year without chemotherapy.

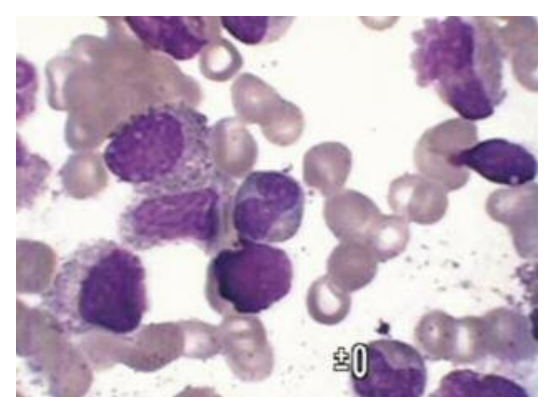

Figure 7. Bone marrow cell morphology examination showing PRCA was stable disease on June 30th, 2015. 
was speculated that this could be the reason PRCA did not progress. Therefore, it is possible that CsA become an indispensable ancillary drug during chemotherapy treatment to prevent relapse and reversing chemoresistance. However, more research needs to be carried out, especially in the form of clinical studies, to confirm the results of the in vitro experiments.

\section{Acknowledgements}

This work was supported by National Natural Science Foundation of China (81071758); Shandong Sciᄀence and Technology Development Project (2015GSF118142); Shandong Medicine and Health Science Technology Program (2016WS0706); China International Medical Foundation (Z20140615325); Natural Science Foundation of Shandong Province Joint Programme (ZR2016HL26, ZR2015HL069); and YantaiYuhuangding Hospital Initiative Foun $\neg$ dation for Young Scientist (201526, 201402); Yantai Science and Technology Program (2016WS013, 2015WS018, 1605350099).

\section{References}

1 Kwong YL, Wong KF, Liang RH, Chu YC, Chan LC and Chan TK: Pure red cell aplasia: clinical features and treatment results in 16 cases. Ann Hematol 72: 137-140, 1996.

2 Murakawa T, Nakajima J, Sato H, Tanaka M, Takamoto S and Fukayama M: Thymoma associated with pure red-cell aplasia: clinical features and prognosis. Asian Cardiovasc Thorac Ann 10: 150-154, 2002.

3 Clark DA, Dessypris EN and Krantz SB: Studies on pure red cell aplasia. XI. Results of immunosuppressive treatment of 37 patients. Blood 63: 277-286, 1984.

4 Chen YT, Feng B and Chen LB: Update of research on drug resistance in small cell lung cancer chemotherapy. Asian Pac J Cancer Prev 13: 3577-3581, 2012.

5 Shen F, Chu S, Bence AK, Bailey B, Xue X, Erickson PA, Montrose MH, Beck WT and Erickson LC: Quantitation of doxorubicin uptake, efflux, and modulation of multidrug resistance (MDR) in MDR human cancer cells. J Pharmacol Exp Ther 324: 95-102, 2008.
6 Mullen B and Richardson JD: Primary anterior mediastinal tumors in children and adults. Ann Thorac Surg 42: 338-345, 1986.

7 Thompson CA: Pure red cell aplasia and thymoma. J Thorac Oncol 2: 263-264, 2007.

8 Chen J, Yang Y, Zhu D, Chen G, Wei S, Qiu X and Zhou Q: Thymoma with pure red cell aplasia and Good's syndrome. Ann Thorac Surg 91: 1620-1622, 2011.

9 Lin CS, Yu YB, Hsu HS, Chou TY, Hsu WH and Huang BS: Pure red cell aplasia and hypogammaglobulinemia in a patient with thymoma. J Chin Med Assoc 72: 34-38, 2009.

10 Nakahara C, Nakamura K, Yamanaka N, Baba E, Wada M, Matsunaga H, Noshiro H, Tanaka M, Morisaki T and Katano M: Cyclosporin-A enhances docetaxel-induced apoptosis through inhibition of nuclear factor-kappaB activation in human gastric carcinoma cells. Clin Cancer Res 9: 5409-5416, 2003.

11 Twentyman PR: A possible role for cyclosporins in cancer chemotherapy. Anticancer Res 8: 985-993, 1988.

12 Goldberg H, Ling V, Wong PY and Skorecki K: Reduced cyclosporin accumulation in multidrug-resistant cells. Biochem Biophys Res Commun 152: 552-558, 1988.

13 Mutch DG, Herzog TJ, Chen CA and Collins JL: The effects of cyclosporin A on the lysis of ovarian cancer cells by cisplatin or adriamycin. Gynecol Oncol 47: 28-33, 1992.

14 Morjani $\mathrm{H}$ and Madoulet C: Immunosuppressors as multidrug resistance reversal agents. Methods Mol Biol 596: 433-446, 2010.

15 Morgan RJ Jr., Synold TW, Gandara D, Muggia F, Scudder S, Reed E, Margolin K, Raschko J, Leong L, Shibata S, Tetef M, Vasilev S, McGonigle K, Longmate J, Yen Y, Chow W, Somlo G, Carroll M and Doroshow JH: Phase II trial of carboplatin and infusional cyclosporine with alpha-interferon in recurrent ovarian cancer: a California Cancer Consortium Trial. Int J Gynecol Cancer 17: 373-378, 2007. 